\title{
Model-Independent Searches for New Physics in Multi-Body Invariant Masses
}

\author{
Sergei Chekanov $^{1, *(\mathbb{D})}$, Smita Darmora ${ }^{1, * \mathbb{D}}$, Wasikul Islam ${ }^{2,3} \mathbb{D}$, Carlos E. M. Wagner ${ }^{1,4} \mathbb{D}^{\mathbb{D}}$ and Jinlong Zhang ${ }^{1}$ \\ 1 HEP Division, Argonne National Laboratory, 9700 S. Cass Avenue, Lemont, IL 60439, USA; \\ cwagner@anl.gov (C.E.M.W.); zhangjl@anl.gov (J.Z.) \\ 2 Department of Physics, Oklahoma State University, Stillwater, OK 74078, USA; wasikul.islam@cern.ch \\ 3 Department of Physics, University of Wisconsin, Madison, WI 53706, USA \\ 4 Physics Department, EFI and KICP, University of Chicago, Chicago, IL 60637, USA \\ * Correspondence: chekanov@anl.gov (S.C.); sdarmora@anl.gov (S.D.)
}

Citation: Chekanov, S.; Darmora, S.; Islam, W.; Wagner, C.E.M.; Zhang, J. Model-Independent Searches for New Physics in Multi-Body Invariant Masses. Universe 2021, 7, 333. https://doi.org/10.3390/ universe7090333

Academic Editor: Lei Wu

Received: 28 July 2021

Accepted: 31 August 2021

Published: 8 September 2021

Publisher's Note: MDPI stays neutral with regard to jurisdictional claims in published maps and institutional affiliations.

\begin{abstract}
Model-independent searches for physics beyond the Standard Model typically focus on invariant masses of two objects (jets, leptons or photons). In this study, we explore opportunities for similar model-agnostic searches in multi-body invariant masses. In particular, we focus on the situations in which new physics can be observed in a model-independent way in three and four-body invariant masses of jets and leptons. Such searches may have good prospects in finding new physics in the situations when two-body invariant masses, which have been extensively explored at collider experiments in the past, cannot provide sufficient signatures for experimental observations.
\end{abstract}

Keywords: beyond the Standard Model (BSM); invariant mass; jets; leptons; large hadron collider (LHC)

Key Contribution: Model-independent searches in invariant masses in collider experiments.

\section{Introduction}

In spite of the great effort put into the search for new physics at the Large Hadron Collider (LHC), no signs of physics beyond the Standard Model (BSM) have yet emerged. If BSM physics exists within the reach of the LHC, it is possible that its experimental signatures are more complicated than originally anticipated. A popular method of finding new physics is to study signal-like deviations in two-body invariant masses of jets, leptons or photons, or studies of event rates, assuming that the Standard Model (SM) background rates are well understood in terms of Monte Carlo (MC) simulations or data-driven control regions. However, it is possible that new physics is hidden in complex event signatures, beyond single- and two-particle distributions for which $\mathrm{MC}$ simulations may not be reliable and/or have significant uncertainties.

In particle collision experiments, model-independent searches for signal-like deviations in invariant-masses distributions of jets, leptons or photons are typically performed by establishing a background hypothesis representing our best knowledge of the SM. Such expectations can be obtained without MC simulations using data-driven "control regions"; i.e., regions of actual data with similar kinematic features but without BSM events. An alternative approach to the description of shapes of the SM background distribution is to perform a fit of the entire mass spectra with some analytic function or using numeric smoothing techniques. After the background hypothesis is established, searches for BSM physics are performed by looking for deviations above the established background shape. Previous searches for heavy resonances in dijet mass distributions $\left(m_{j j}\right)$ using the techniques described above have been performed by ATLAS [1,2] and CMS [3-5].

Model-independent searches using the above-mentioned technique applied to twobody invariant masses are rather popular at the LHC. Only a handful number of LHC 
studies [6,7] have gone beyond the two-object mass distributions. In particular, searches in multi-body invariant masses that involve different objects (jets, leptons, photons etc.), without relying on MC predictions, are almost non-existent. In this respect, LHC data have not been fully explored in multi-body invariant masses with the same precision as in published papers with two-body masses.

In this paper, we investigate three and four-body invariant masses for BSM processes. We discuss the importance of such studies when direct observations of BSM signals in two-body decays (i.e., dijets, di-leptons or di-photons) are difficult. The latter can be due to their large width or due to significant backgrounds from inclusive multi-jet QCD events. A typical requirement for the partial width $(\Gamma)$ and the mass $(m)$ for a heavy particle leading to signals that can be observed in invariant masses using the model-independent approach is $\Gamma / m<0.2$. Other possible difficulties with two-body invariant masses are a large SM background or small masses $\left(m_{j j}<0.5 \mathrm{TeV}\right)$ which are difficult to study due to trigger pre-scales [1-5].

Recently, a class of models predicting wide $Z^{\prime}$ resonances that cannot easily be found using the conventional experimental methods based on two-body invariant masses has been discussed in [8,9]. In this paper, we argue that BSM events with similar broad states coupled to SM fermions can be identified using three and four-body decays.

\section{Final State Radiation in BSM Models}

Due to a low trigger efficiency for jets with momenta below about $0.5 \mathrm{TeV}$, two-jet invariant masses are difficult to describe at the LHC using analytic functions or smoothing techniques. As the result of such difficulties, many experimental searches focus on highmass regions (typically, above $1 \mathrm{TeV}$ ). To mitigate the trigger problem for low-mass searches, one can require an associated object that can be used for event triggering. This object can be a photon $[10,11]$ or a jet [12-15] from initial-state radiations.

Particles such as photons and vector-bosons from the final-state radiation of quarks in the decays such as $Z^{\prime} \rightarrow q \bar{q}$ are a valuable option for searches for new physics in twobody invariant masses. In such cases, additional objects from final-state radiation, such as photons or leptons (from $W / Z$ ), can be used for both event triggering and for calculations of multi-body invariant masses used in searches. The rate of such events is expected to be significantly lower than that in the inclusive dijet case. The main benefit of such events is in the smoothness of the expected background shape for low masses and the existence of a statistically independent event sample ${ }^{1}$ to construct control regions. Experimentally, the inclusion of additional electromagnetic objects (such as leptons $l$ or photons $\gamma$ ) to a dijet invariant mass should not significantly increase the resolution of the reconstructed invariant masses. Therefore, the searches in the masses such as $m_{j j \gamma}$ or $m_{j j l}$ may have advantages for low invariant-mass studies and at least should be considered on an equal footing with the dijet studies.

\section{Cascade Decays of BSM Particles}

Let us consider several event topologies that have a special property for modelindependent BSM searches: such events might be difficult to observe in two-body decays, but many-body decays can exhibit signals that can be detected through observations. To be more concrete, let us consider a cascade decay of a heavy particle $A$ into two other particles $B$ and $C$. Such decays are typical for the diboson production (for a recent review, see [16]). The most popular channel in experimental searches is when $B$ and $C$ are known bosons $(W$, $Z$ or $H$, on-shell or off-shell). Generally, however, $A$ and $B$ may not be known. In the case when $B$ and $C$ are bosons, their two-body decay modes are ether hadronic $(q \bar{q})$ or leptonic $\left(l^{ \pm} v, l^{+} l^{-}\right)$. To be more specific, we assume that $B$ decays to two jets, while $C$ decays to a lepton and $X$, where $X$ is another lepton, neutrino or some other particle (detectable or undetectable). This situation is depicted in Figure 1, where the dashed line from the particle $C$ represents such an additional particle. 


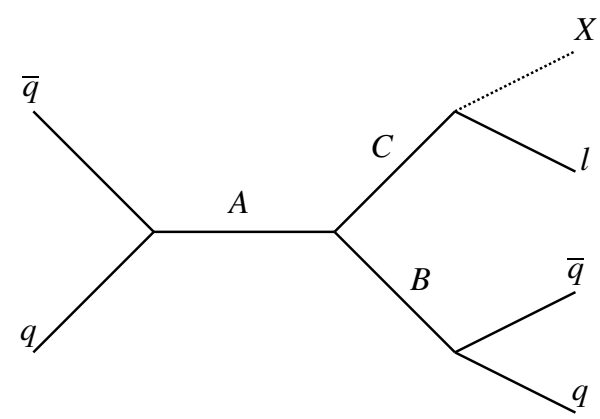

Figure 1. A schematic representation of the decay a heavy particle $A$ to two other particles, $B$ and $C$.

Searches involving jets and a lepton in the final state that originate from the cascade decays shown in Figure 1 were recently studied [17] by ATLAS using a model-independent approach by triggering on leptons (electrons or muons) and by performing searches in invariant masses, $m_{j j}$, of two jets. A limitation of such an approach is the width of the heavy particle $B:$ if $\Gamma / m>0.2$, the studies show a small sensitivity to such broad states since such deviations are almost indistinguishable from the background hypothesis.

The studies [17] can be extended by allowing a lepton $l$ to participate in the reconstruction of invariant masses. In this case, the three-body invariant masses, $m_{j j l}$, can be calculated and studied in exactly the same way as the dijet masses $m_{j j}$. In the situations in which the original particle $A$ has a small partial width, the three-body decays can exhibit a narrow signal, which can be sufficient for the observation of this class of BSM processes. Thus, even though particle $B$ is not directly observable in dijet decays due to its wide width, the original particle $A$ can still be found by observing a deviation on a smooth background distribution using a model-independent approach based on a global fit (or a numeric smoothing technique). Such a class of physics studies has not been extensively explored at the LHC. Below, we illustrate that the expected SM background for three-body decays is indeed a smoothly falling distribution.

\subsection{Monte Carlo Simulations}

In this section, we illustrate several physics scenarios where three and four-body invariant masses can be a useful tool for observing new physics. For our studies, we use the PYTHIA 8 MC model $[18,19]$ for the generation of $p p$-collision events at $\sqrt{s}=13 \mathrm{TeV}$ center of mass energy. Effects from double parton scattering are not included in the simulation. The NNPDF 2.3 LO [20] parton density function from the LHAPDF library [21] was used. Jets, isolated electrons and muons were reconstructed from stable particles. Jets were constructed with the anti- $k_{T}$ algorithm [22] as implemented in the FASTJET package [23] using a distance parameter of $R=0.4$. The minimum transverse energy of all jets was $40 \mathrm{GeV}$ in the pseudo-rapidity range of $|\eta|<2.5$. The leptons are required to be isolated using a cone of the size 0.2 in the azimuthal angle and pseudo-rapidity defined around the true direction of the lepton. All energies of particles inside this cone are summed. A lepton is considered to be isolated if it carries more than $90 \%$ of the cone energy. All other details of simulation and reconstruction are described in [29].

\subsection{Three-Body Invariant Masses}

Let us discuss experimental situations in which three-body invariant masses can be more useful than the usual two-body masses. Three-body invariant masses $m_{j j l}$ reconstructed from two jets and a lepton can be a more powerful variable in the identification of the process $A \rightarrow B C$ than two-body masses under the following conditions:

- Two jets from decays of the particle $B$ should be resolved using the standard jet algorithms; see Figure 1. The angular separation of two jets can be approximated by $2 m_{B} / p_{T}^{B}$. This implies that the mass of $B$ should be comparable with the mass of $A$, such that the particle $B$ will not receive a significant boost in transverse momentum; thus, the two jets from the particle $B$ can be well resolved. 
- The presence of at least one lepton in an event does not set any particular constraints on the mass of $C$ compared to the mass of $A$. For example, the decay of particle $C$ can be considered fully boosted; i.e., two leptons originating from $C$ will be merged into a single lepton, as seen by experiments. This can happen when the particle $C$ has a significantly smaller mass compared to $A$; thus, the particle $C$ receives a significant boost in momentum. For TeV scale searches of the particle $A$, the SM $Z$ boson with the fully boosted decay to $l^{+} l^{-}$can be a possible candidate for $C$.

There is another option for this topology: $C \rightarrow l+X$, where $X$ is an undetectable particle, as shown in Figure 1. The particle $C$ can be a $W$ boson, or a BSM boson-like particle, while $X$ can be a neutrino. Such cases also do not impose any restrictions on the mass of $C$ relative to the mass of $A$.

The above scenarios are convenient for model-independent searches using a datadefined (or phenomenological) smoothly-falling shape for the $m_{j j l}$ distribution if the partial width of $A$ is narrow; i.e., $\Gamma / m<0.2$. The advantage of searches in $m_{j j l}$, compared to the direct observation of $B$ in the dijet masses $m_{j j}$, is most obvious in the case when the partial width of $B$ is large (as in the case of strong decays).

Another possible combination for three-body invariant masses is $j+l l$. If a particle $C$ decays to two resolved leptons, then this type of decay should be detected via the direct searches in di-lepton channels. Thus, this channel should be well covered by the existing LHC studies. On the other hand, if $C$ decays to a highly boosted di-lepton pair, which is resolved as a single lepton, and particle $B$ decays to a jet and lepton, this study can be partially covered by the searches for quantum black holes [25] that use the $m_{j l}$ invariant masses.

\section{Specific BSM Models}

In this section, we consider several specific BSM models that can be found using three-body invariant masses.

\subsection{Models with SM Bosons}

A cascade decay of two heavy particles with the associated $W$ gauge boson decaying to $l v$ is the natural class of events where multi-body invariant masses are useful for BSM searches. A generic diagram of such processes is shown in Figure 2.

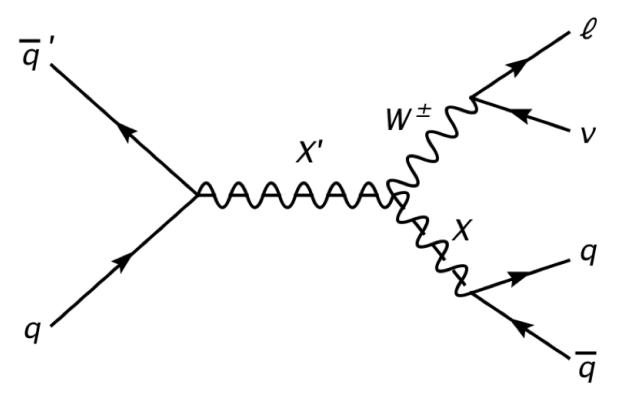

Figure 2. A representative Feynman diagram for a generic resonance, $X$, decaying to two partons in association with a leptonically decaying $W$ boson in the $s$-channel. The latter channel includes a resonance $X^{\prime}$ decaying to a $W$ and an $X$.

As an example, let us consider the processes $W^{\prime} \rightarrow W Z^{\prime} \rightarrow \ell v q \bar{q}$ and the $\rho_{T} \rightarrow$ $W^{ \pm} \pi_{T} \rightarrow \ell v q \bar{q}$ process discussed in [17]. The $W^{\prime} \rightarrow W Z^{\prime} \rightarrow \ell v q \bar{q}$ and $\rho_{T} \rightarrow W^{ \pm} \pi_{T} \rightarrow$ $\ell v q \bar{q}$ simulations assume a $Z^{\prime}$ dijet resonance produced in association with a leptonically decaying $W$ from the $q \bar{q} \rightarrow W^{\prime}$ process. The mass difference between the $W^{\prime}$ and $Z^{\prime}$ was set to $250 \mathrm{GeV}$. The latter requirement yields the largest predicted cross-section for the desired final state [17].

The second process discussed in [17] is $\rho_{T} \rightarrow W^{ \pm} \pi_{T} \rightarrow \ell v q \bar{q}$. It is a generic technicolor model with a technirho, $\rho_{T}$, that decays into a leptonically decaying $W$ boson and a 
technipion $\pi_{T}$, decaying into two jets. The mass of the $\rho_{T}$ is chosen to be larger than the mass of the $\pi_{T}$ by a factor of two, which maximizes the cross-section for the $l v q \bar{q}$ final state. These processes are quite distinct kinetically: in one case, the phase space for $W$ production is limited, while it increases with the mass of $\rho_{T}$ for the technicolor model.

Figure 3 shows the two-jet and two-jet plus lepton invariant masses for the processes discussed above. The processes were simulated and reconstructed as discussed in Section 3. The result shows that there is no principal difference in the width of the two-jet and threebody invariant mass for $W^{\prime} \rightarrow W Z^{\prime} \rightarrow \ell v q \bar{q}$. The relative widths of these masses are below $11 \%$ (this was estimated by taking the $90 \%$ root-mean squared (RMS) value of the distribution and dividing by the mass). Thus, the effect $\mathrm{f}$ the missing neutrino is small. This is not the case for $\rho_{T} \rightarrow W^{ \pm} \pi_{T} \rightarrow \ell v q \bar{q}$, where the width of the three-body mass is significantly larger than that for the two-jet mass.

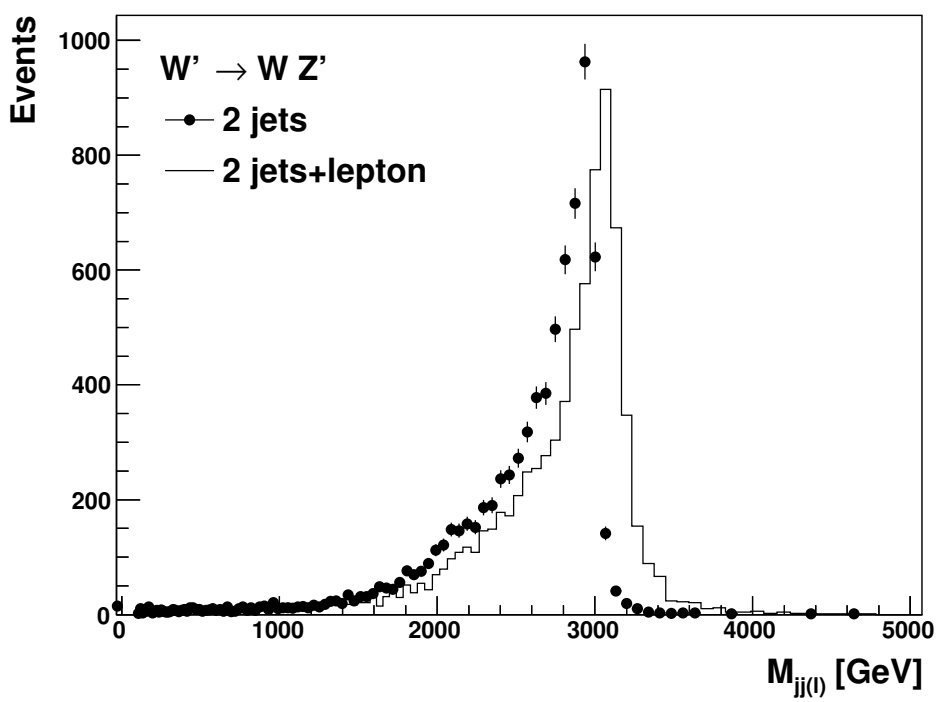

(a) $W^{\prime} / Z^{\prime}$ SSM model

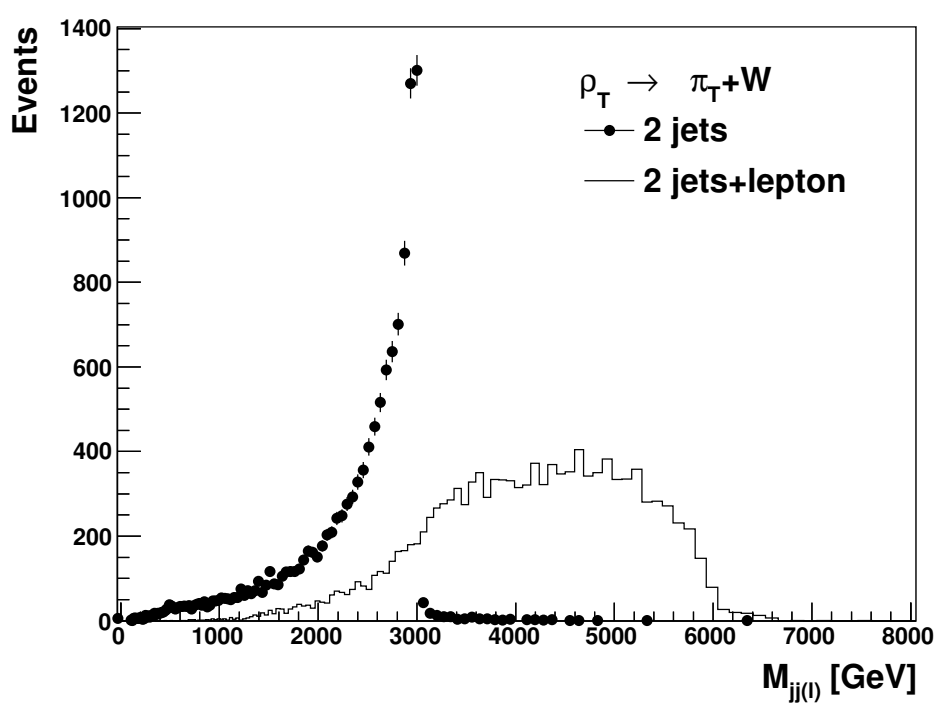

(b) $\rho_{T}$ model

Figure 3. The invariant masses of two jets $\left(m_{j j}\right)$ and two jets and a lepton $\left(m_{j j l}\right)$ for $(\mathbf{a}) W^{\prime} \rightarrow W Z^{\prime} \rightarrow$ $\ell v q \bar{q}$ and (b) $\rho_{T} \rightarrow W^{ \pm} \pi_{T} \rightarrow \ell v q \bar{q}$ process discussed in [17]. The SSM process has a mass splitting between $W^{\prime}$ and $Z^{\prime}$ of $250 \mathrm{GeV}$. The second model has a mass of the $\rho_{T}$ that is twice as large as that of the $\pi_{T}$.

These examples show that, in some scenarios, such as the first one discussed above, the discovery potential using three-body masses may be as strong as when using the 
two-jet masses. Such scenarios belong to the cases when the phase space for production of additional particles, such as $W$, is restricted, and the contribution from the missing neutrino can be approximately ignored. In the unrestricted case, instead, the inclusion of missing energy (MET) from escaping neutrinos leads to broad mass distributions, thus preventing an effective search in smoothly decreasing distributions. Such broad distributions are due to the fact that the reconstructed transverse mass distribution is, in general, much wider than the invariant mass.

\subsection{Radion Models}

Generally, in order to identify a scenario in which three-body masses have a strong potential for narrow signal signatures, a scan of free parameters needs to be performed. The main tunable parameter is the mass splitting between the two heavy BSM particles. As an example of this scenario, let us consider the following model [26]:

$$
W_{k k} \rightarrow W+\varphi \rightarrow l v+g g
$$

where $W_{k k}$ denotes the Kaluza-Klein excitation ("KK boson") and $\varphi$ is a radion particle decaying to two gluons (i.e., jets). Since the $W$ bosons decay into $l v$, the events should include a $v$ leading to missing transverse energy (MET). Adding reconstructed MET to the invariant mass reconstruction significantly increases the observed signal width. Therefore, as in the first example in the last section, we explore physics scenarios in which MET is not required (i.e., when the transverse energy of $v$ is not large).

In this study, we varied the masses of $W_{k k}$ and $\varphi$ and found a case when the width of the three-body invariant mass is the same as in the case of two-body decays (corresponding to two-jets from the radion). Figure 4 a shows the scenario in which the difference in masses between $W_{k k}$ and $\varphi$ is $250 \mathrm{GeV}$; i.e., $M_{W_{k k}}-M_{\varphi}=250 \mathrm{GeV}$. This leads to the width of $m_{j j l}$ being close to the width of $m_{j j}$.

Another alternative scenario is shown in Figure $4 \mathrm{~b}$, where $M_{\varphi}=M_{W_{k k}} / 2$. In the latter case, MET should not be ignored since the neutrino has a significant transverse momentum. It should be noted that, at large $W_{k k}$ masses, the cross-section for the second scenario shown in Figure $4 \mathrm{~b}$ is a factor $2-5$ larger than for the first scenario. However, the benefit of having a narrow signal width for $m_{j j l}$, leading to large reconstruction sensitivity over a smooth SM background, can compensate for the effects of a lower absolute cross-section. Therefore, the scenario with mass splitting between the two BSM particles close to the mass of associated particles (such as $\mathrm{W} / \mathrm{Z} / \mathrm{H}$ ) is a preferable configuration for experimental searches in three-body decays.

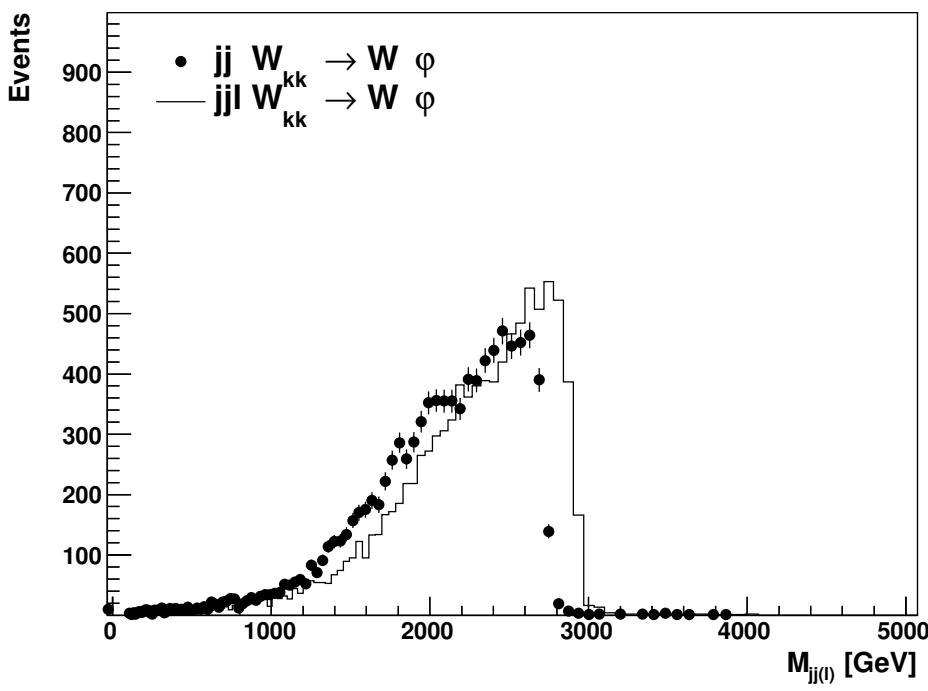

(a) Case 1

Figure 4. Cont. 


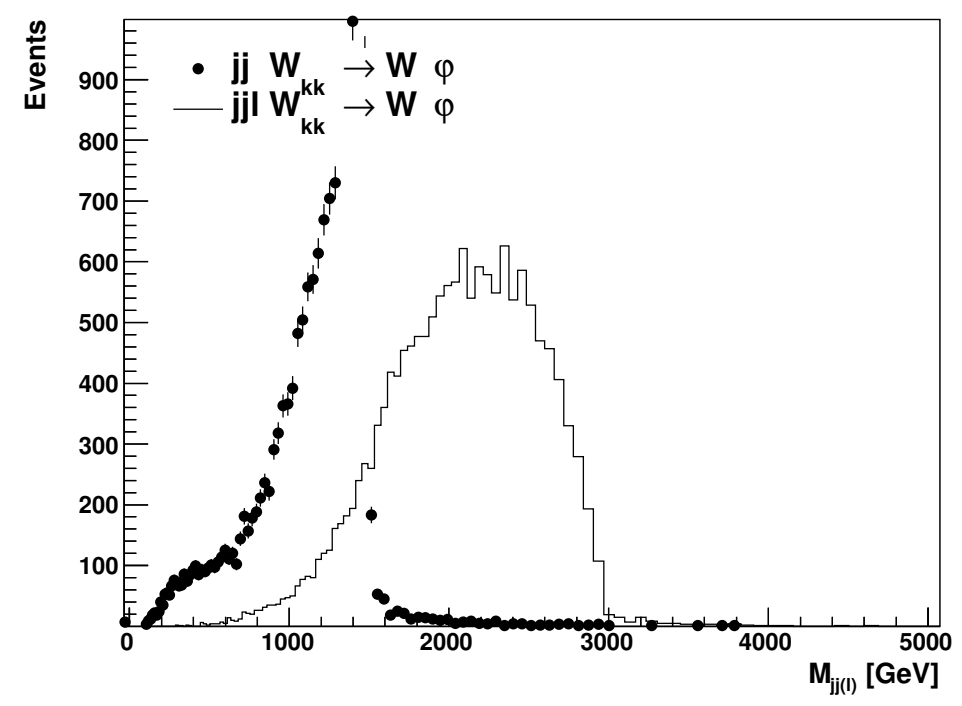

(b) Case 2

Figure 4. The $m_{j j}$ and $m_{j j l}$ invariant mass distributions for the $3 \mathrm{TeV} W k k$ mass when (a) case 1 : $M_{W_{k k}}-M_{\varphi}=250 \mathrm{GeV} \&(\mathbf{b})$. Case $2: M_{\varphi}=M_{W_{k k}} / 2$.

\subsection{Composite Resonances}

Among different composite resonances models that break lepton flavor (LF) universality, the decay channel $p p \rightarrow V \rightarrow E^{ \pm} l^{\mp}$ (where $E$ is a heavy composite lepton which decays into SM bosons and leptons $E \rightarrow Z / h+l$ ) [27] can easily be probed using the multi-body invariant masses. Figure 5 shows a Feynman diagram for a model where $V$ is a heavy $Z^{\prime}$ boson $\left(M\left(Z^{\prime}\right)>M(E)\right)$. When $Z / h$ decays hadronically, invariant masses of two jets and a lepton $\left(m_{j j l}\right)$ can be used to reconstruct the mass of $E$. In addition, the $2 \mathrm{j}+21$ mass $\left(m_{j j l l}\right)$ is sensitive to the $V\left(Z^{\prime}\right)$ mass itself. It should be noted that even if the partial width of the composite $E$ is broad (and thus $m_{j j l}$ is not sensitive to the presence of $E$ ), the invariant mass $m_{j j l l}$ can still be narrow if the partial width of $V$ is small (which is a typical case for $Z^{\prime}$ ).

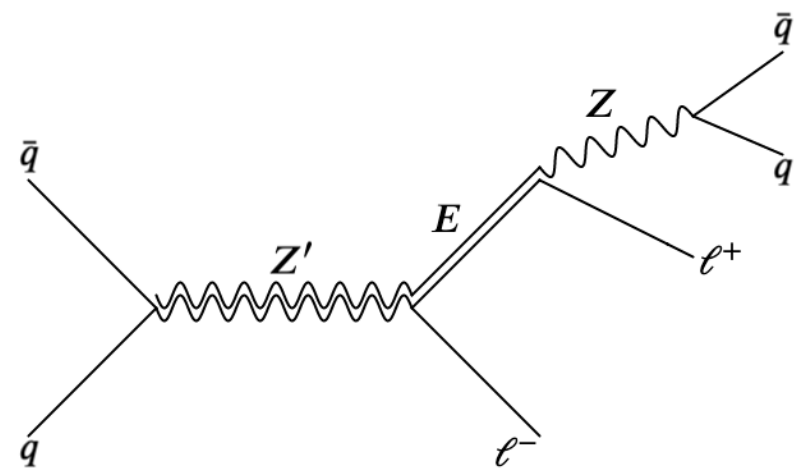

Figure 5. A representative Feynman diagram for the composite lepton model (see Section IV of [27]) where $m_{j j l}$ and $m_{j j l l}$ can be used for searches of $V=Z^{\prime}$ and a composite lepton-like particle $E$.

Figure 6 shows the $m_{j j}, m_{j j l}$ and $m_{j j l}$ invariant masses reconstructed at the truth level of a Monte Carlo simulation. The selection cuts are set as for the default analysis used in this study. The simulations are performed using Madgraph5 (MG5) [28] with the PYTHIA 8 shower. The masses are set to $M\left(Z^{\prime}\right)=3 \mathrm{TeV}$ and $M(E)=2 \mathrm{TeV}$ in the MG5 simulation. The observed peak near $3 \mathrm{TeV}$ (shown with dotted line) is due to the decay of $Z^{\prime}$. The solid line shows two peaks. The largest peak is a reflection of the $E$ decays, while the smaller peak is a reflection of the $Z^{\prime}$ decays. The two-jet masses show the mass peak at the $Z$ mass, but it has a more complicated distribution than multi-body decays. This figure illustrates that both $2 j+1$ and $2 j+2 l$ invariant masses can be used for searches of events originating from the composite resonances models [27]. 


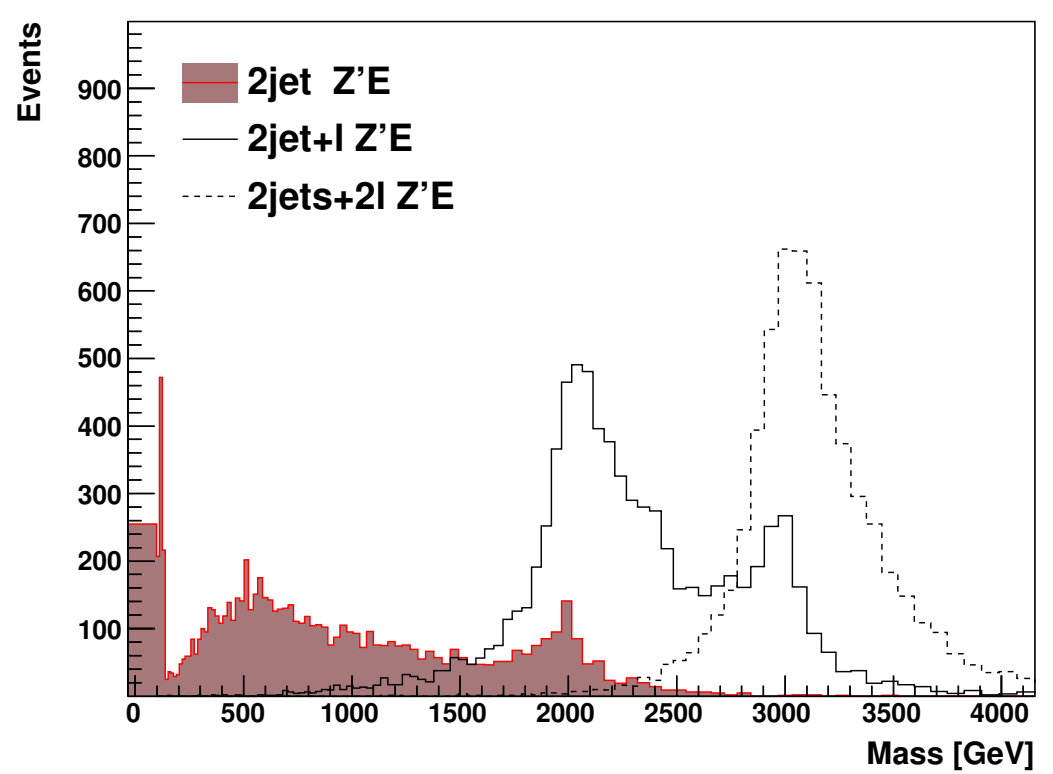

Figure 6. Invariant masses for a $Z^{\prime} E$ model [27] constructed from two jets, two jets plus a lepton and two jets plus two leptons. The $Z^{\prime}$ mass was generated at $3 \mathrm{TeV}$, while $M(E)=2 \mathrm{TeV}$. The simulations were performed using MG5 with PYTHIA 8 showering.

\subsection{Hypothetical Scenarios}

Based on our analyses in the previous sections, let us consider hypothetical examples in which two-body invariant masses are at a disadvantage for searches since the two-body signal width is larger than three or four-body masses. The goal of such simulation is to illustrate a possible parameter space when two-body masses are less powerful for searching for enhancements on a smoothly falling background than for multi-body masses.

\subsubsection{Three-Body Invariant Masses}

For the three-body example, we set up a PYTHIA 8 simulations for the process $W^{\prime} \rightarrow Z^{\prime} W^{*}$, where $W^{\prime}, Z^{\prime}$ and $W^{*}$ are hypothetical bosons, without assuming any particular physics couplings or model. In this simulation, the mass of $W^{\prime}$ was set to $1 \mathrm{TeV}$ and its width was set to $\Gamma=10 \mathrm{GeV}$. $Z^{\prime}$ decays to two jets, while $W^{*}$ decays to a lepton and a neutrino. The mass of $Z^{\prime}$ was set to $500 \mathrm{GeV}$ (with $\Gamma=250 \mathrm{GeV}$ ), and the mass of $W^{*}$ was set to $300 \mathrm{GeV}$ (with $\Gamma=250 \mathrm{GeV}$ ). The widths of $Z^{\prime}$ and $W^{*}$ in this setup are large $(\Gamma / m \geq 0.5)$, and the detection of such particles via the observations of Gaussian-shaped enhancements on smoothly falling $m_{j j}$ distributions is difficult. However, for the $m_{j j l}$ masses, the situation is different. Figure 7 shows that the width of $m_{j j l}$ is smaller than the width of $m_{j j}$ despite the presence of the neutrino that introduces a shift and smearing in the mass distribution. According to this simulation, the relative width of the $m_{j j l}$ masses is close to $15 \%$, which is in the range of acceptable width for the experimental detection on smoothly falling background distributions. 


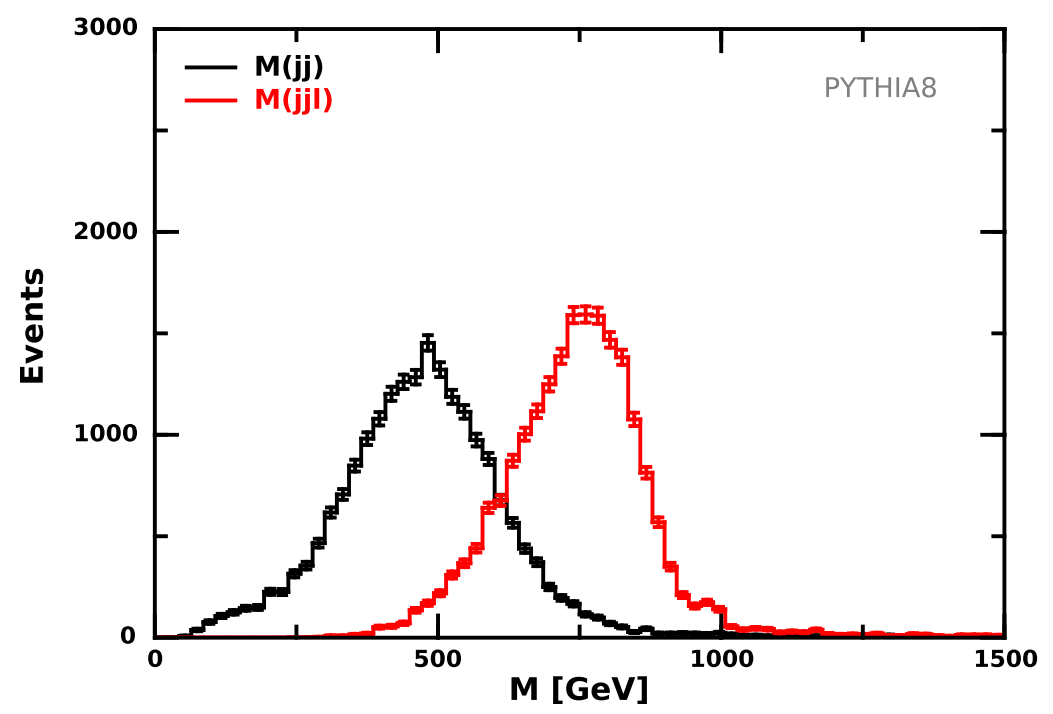

Figure 7. The invariant masses of two jets $\left(m_{j j}\right)$ and two jets and a lepton $\left(m_{j j l}\right)$ in events $A \rightarrow B C$ simulated with PYTHIA 8 with the settings described in the text.

\subsubsection{Four-Body Invariant Masses}

For the four-body decay analysis, consider the example shown in Figure 1, where $X$ could be a lepton or a jet. Hence, events with at least two jets and leptons lead to the following combinations: $m_{j j l}$ and $m_{j j j l}$. As in the case of three-body decays, modelindependent searches for signal-like enhancements in smoothly falling distributions of four-body invariant masses can be simpler than for similar searches in two-body decays when the partial widths of $B$ and $C$ have $\Gamma / m>0.2$.

Here are possible kinematic scenarios that are not well covered in $p p$ collider experiments:

- $\quad$ Signature searches based on $m_{j j l}$ invariant masses for discovering a TeV-scale particle decaying to two heavy particles when both $B$ and $C$ are heavy; thus, there is no significant momentum boost, and all decay products are well resolved.

- Event topologies contributing to $m_{j j j l}$, when a particle $C$ decays to a lepton and jets, similar to quantum black hole scenarios [25].

Let us consider a MC simulation for the first scenario. We have created the PYTHIA 8 simulation for the process $W^{\prime} \rightarrow Z^{\prime} W^{*}$, where $W^{\prime}, Z^{\prime}$ and $W^{*}$ are hypothetical bosons. The mass of $W^{\prime}$ was set to $1 \mathrm{TeV}$ and the width $\Gamma=10 \mathrm{GeV}$. $Z^{\prime}$ decays to two leptons (electrons or muons), while $W^{*}$ decays to two jets. The mass of $Z^{\prime}$ was set to $500 \mathrm{GeV}$ (with the width of $250 \mathrm{GeV}$ ), and the mass of $W^{*}$ was set to $300 \mathrm{GeV}$ (with the width of $150 \mathrm{GeV}$ ). Thus, the widths of $Z^{\prime}$ and $W^{*}$ are broad, and a detection of such particles via the observations of Gaussian-shaped enhancements in two-body decays is difficult. Figure 8 shows that the relative width of $m_{j j l}$ is smaller than the widths of two-body decays of $Z^{\prime}$ and $W^{*}$. Thus, the relative width of the $m_{j j l}$ distribution due to the $W^{\prime}$ decays is close to the range for a direct observation of such a state over a smoothly falling background. 


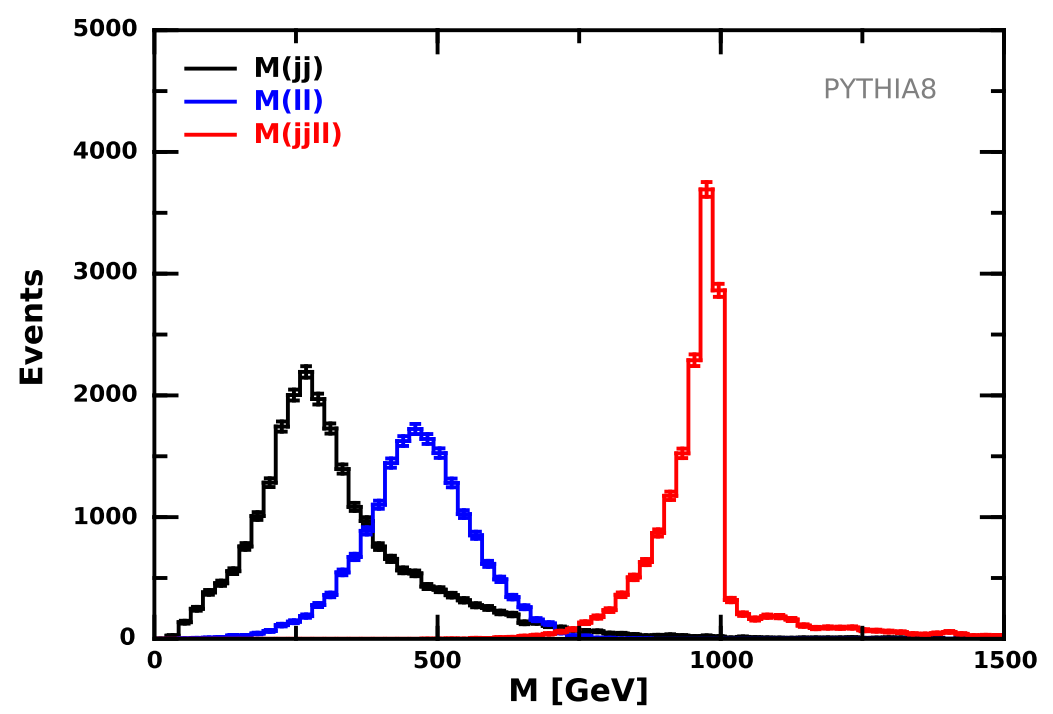

Figure 8. The invariant masses of two jets $\left(m_{j j}\right)$, two leptons $\left(m_{l l}\right)$ and two jets and two leptons $\left(m_{j j l l}\right)$ in events $A \rightarrow B C$ simulated by PYTHIA 8 with the settings as described in the text.

\section{Exclusion Limits}

Although it would be interesting to derive the limits from four-body invariant masses, such as $m_{j j l l}$ or $m_{j j j l}$, obtaining these limits is difficult due to the lack of experimental publications that can be used to correct Monte Carlo predictions for realistic lepton misidentification rates.

To set upper limits on the observation of a new resonance in the $m_{j i l}$ invariant mass, Monte Carlo simulations have been performed using PYTHIA 8 with settings and object reconstruction as described in Section 3.1. Three SM processes were generated: (1) lightflavor QCD dijets, (2) vector and scalar boson production and (3) $t \bar{t}$. Pileup events were not simulated, but this problem is mitigated by using the normalization factors described below. All other details in the simulation match the description of [24]. Unlike the signal models, the SM background processes require simulations of misidentification rates for muons and leptons ("fake rates"). We used a $0.1 \%$ misidentification rate for muons and $1 \%$ fake rate for electrons [24]. This was implemented by assigning the probability of $10^{-3}$ $\left(10^{-2}\right)$ for a jet to be identified as a muon (electron) using a random number generator. The distributions were obtained for events with at least one isolated lepton with $p_{T}^{l}>60 \mathrm{GeV}$ and two jets at $p_{T}>30 \mathrm{GeV}$. The SM background MC samples are available from the HepSim repository [29].

After the $m_{j j l}$ distributions were reconstructed assuming $140 \mathrm{fb}^{-1}$ separately for muons and electrons, they were scaled to match the official ATLAS Monte Carlo [17]. ${ }^{2}$ This corrected for residual differences between the truth-level simulations as described above and the ATLAS simulations that included realistic detector effects; i.e., $m_{j j l}$-dependent lepton misidentification rates and pile-up contributions. The values of the correction were between 2 and 3 , depending on $m_{j j l}$. These values were almost entirely attributed to the correction for the lepton misidentification rates.

Figure 9 shows the invariant masses using three luminosity scenarios: $140 \mathrm{fb}^{-1}$, $440 \mathrm{fb}^{-1}$ (LHC Run 2+ Run 3) and the HL-LHC ( $3 \mathrm{ab}^{-1}$ for $14 \mathrm{TeV}$ ). The latter distribution was corrected by a correction factor of $12 \%$ (estimated using PYTHIA 8 ) to reflect the change in the CM energy from 13 to $14 \mathrm{TeV}$. The number of entries per bin was divided by the bin widths. The distributions have smoothly falling shapes, similar to those for the two-body invariant masses studied by ATLAS [1,2] and CMS [3-5]. Therefore, the $m_{j j l}$ distributions can be examined for local excesses above a data-derived estimate of the smoothly falling predictions, which can be obtained using various smoothing techniques or by performing a global fit with an analytic function. 


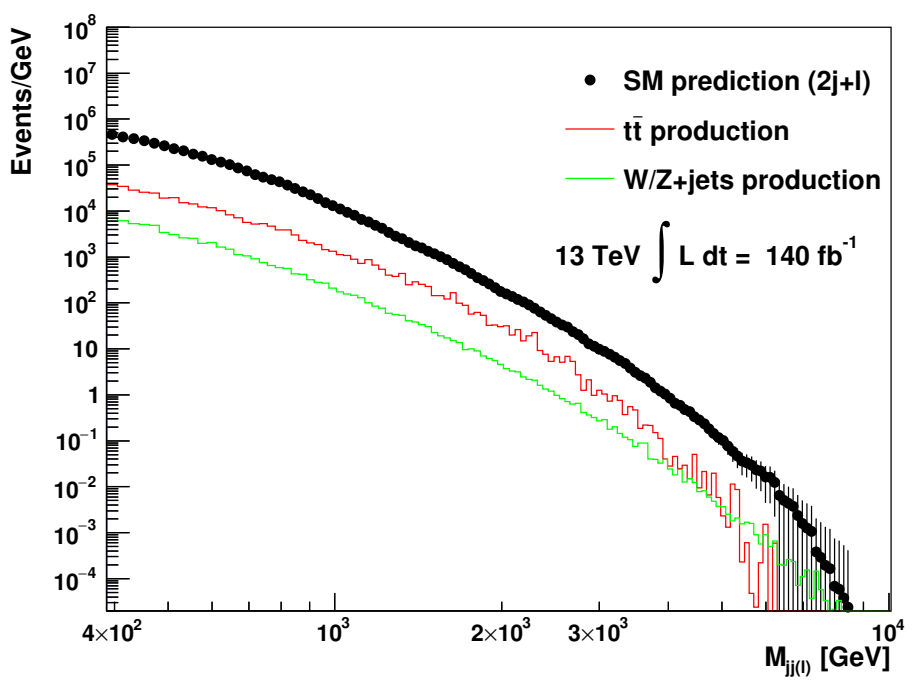

(a) $140 \mathrm{fb}^{-1}$

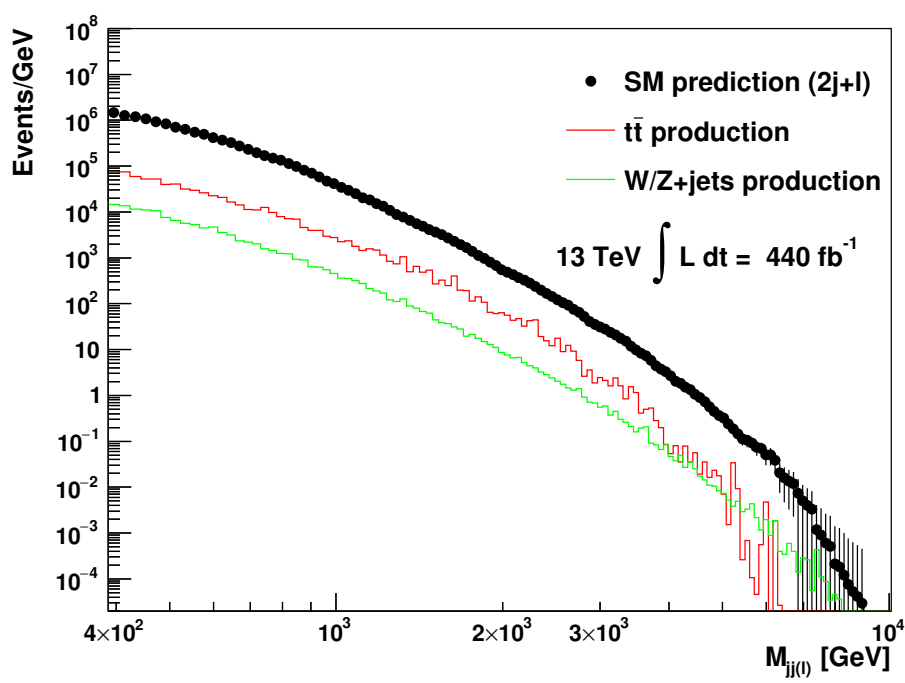

(b) $440 \mathrm{fb}^{-1}$

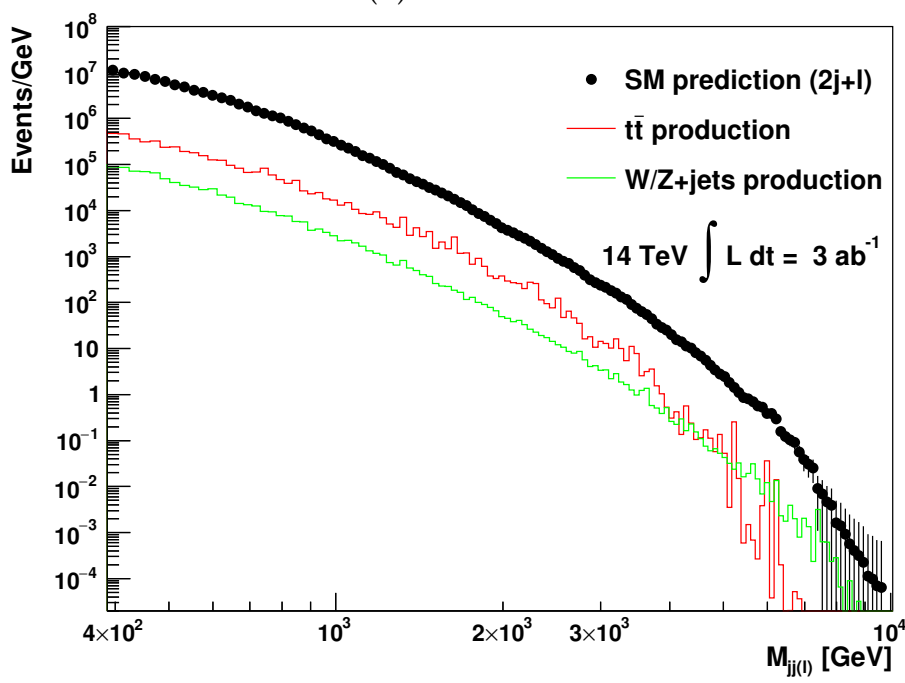

(c) $3 a b^{-1}$

Figure 9. Expectations for $m_{j j l}$ invariant mass distributions for $140 \mathrm{fb}^{-1}, 440 \mathrm{fb}^{-1}$ and $3 \mathrm{ab}^{-1}(14 \mathrm{TeV})$ using the PYTHIA 8 generator for events with at least one isolated lepton with $p_{T}^{l}>60 \mathrm{GeV}$. Contributions from $\mathrm{W} / \mathrm{Z} / \mathrm{H}^{0}$-boson processes and top-quark processes are shown separately (without stacking the histograms). 
Figure 10 shows the expected 95\% confidence level (C.L.) upper limit on the fiducial cross-section times the branching ratio for a generic Gaussian signal with the width $\left(\sigma_{G}\right)$ being $15 \%$ of the Gaussian peak position, while decaying to two jets and a lepton. The results are presented for different values of the integrated luminosity, including the luminosity expected after LHC run 3 and high-luminosity LHC. The limits were calculated using the frequentist approach [30] with the asymptotic approximation ${ }^{3}$. The $95 \%$ quantile of the posterior was taken as the upper limit on the possible number of signal events in data corresponding to that mass point. This value, divided by the corresponding luminosity, provided the upper limit on the production cross-section of a new particle times the branching ratio $(\mathrm{Br})$ to two jets plus a lepton. The presented limits can be used to estimate the sensitivity of the LHC data to any phenomenological models predicting Gaussian signals in the $m_{j j l}$ distributions.

As emphasized above, the limits for the four-body masses, such as $m_{j j l l}$ or $m_{j j j l}$, are difficult to obtain due to the lack of experimental publications that can be used to correct Monte Carlo predictions for realistic lepton misidentification rates.

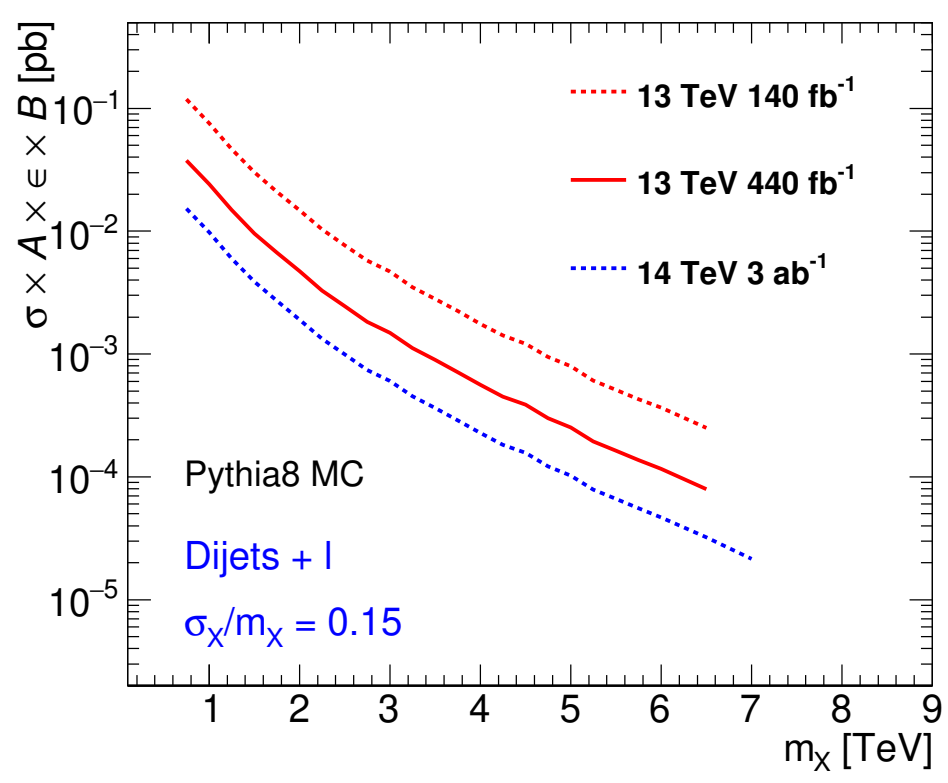

Figure 10. The 95\% C.L. upper limits obtained from the $m_{j j l}$ distribution on cross-section times acceptance $(A)$, efficiency $(\epsilon)$ and branching ratio (BR), for a BSM signal with a cross-section that produces a Gaussian contribution to the particle-level $m_{j j l}$ distribution, as a function of the mean of the Gaussian mass distribution. The limits are calculated for a BSM signal assuming $\sigma_{X} / m_{X}=0.15$ width.

\section{Summary}

This paper discusses several scenarios for model-independent BSM searches that have not received sufficient attention in collider experiments. In particular, we focus on generic processes with cascade decays of the type $A \rightarrow B C \rightarrow j j l(l)$, where $A$ is a heavy BSM particle, while either $B$ or $C$ is another heavy particle decaying to jets with a mass smaller than $A$. For the physics processes discussed in this paper, multi-body invariant masses calculated using two jets and leptons may lead to favorable conditions for experimental observations of signal-like structures on smoothly falling invariant masses. Such structures can be observed with high precision using various smoothing numerical techniques or a global fit of multi-body invariant masses using analytic functions. All methods of this type are widely utilized in dijet searches.

We believe that multi-body final states with leptons are largely unexplored using high-precision model-independent techniques that do not rely on Monte Carlo predictions for SM background. It would be interesting to explore general theoretical scenarios with narrow heavy particles that decay to relatively broad resonances. Such BSM events can easily escape observations using previously published searches in dijets, which are not 
the primary observables for such BSM events. Dijet searches in inclusive collision events are also affected by the overwhelming multi-jet QCD background. Therefore, multi-body invariant masses that include leptons have the potential to bring unexpected discoveries. Using Monte Carlo simulations, our analysis set 95\% credibility-level upper limits on the signal cross-section times acceptance times efficiency times the branching ratio for new processes that can produce a Gaussian contribution to the dijet plus lepton invariant-mass distribution. The estimated limits can be used to guide model builders, who hypothesize that heavy states lead to signal signatures in such multi-body invariant masses.

The approach described in this paper can equally be applied to invariant masses that include more than four objects or invariant masses that are composed of only jets or leptons. However, such studies are beyond the scope of the current paper.

Author Contributions: Conceptualization, S.C.; Formal analysis, S.D.; Methodology, C.E.M.W.; Project administration, J.Z.; Software, S.C. and W.I.; Visualization, W.I.; Writing-original draft, S.C. and S.D.; Writing-review \& editing, S.D. All authors have read and agreed to the published version of the manuscript.

Funding: This research received no external funding.

Institutional Review Board Statement: Not applicable.

Informed Consent Statement: Not applicable.

Data Availability Statement: Data available from the HepSim Monte Carlo repository https:// atlaswww.hep.anl.gov/hepsim/ (accessed on 7 September 2021).

Acknowledgments: We would like to thank K. S. Agashe, H. Meng, P. Du and M. Chala for the discussions of Monte Carlo simulations for the radion and composite BSM models. W. Islam would like to thank A. Khanov also for some useful discussions. We gratefully acknowledge the computing resources provided by the Laboratory Computing Resource Center at Argonne National Laboratory. The submitted manuscript has been created by UChicago Argonne, LLC, Operator of Argonne National Laboratory ("Argonne"). Argonne, a U.S. Department of Energy Office of Science laboratory, is operated under Contract No. DE-AC02-06CH11357. The U.S. Government retains for itself, and others acting on its behalf, a paid-up nonexclusive, irrevocable worldwide license in said article to reproduce, prepare derivative works, distribute copies to the public, and perform publicly and display publicly, by or on behalf of the Government. The Department of Energy will provide public access to these results of federally sponsored research in accordance with the DOE Public Access Plan. http:/ / energy.gov / downloads/doe-public-access-plan (accessed on 7 September 2021). Argonne National Laboratory's work was funded by the U.S. Department of Energy, Office of High Energy Physics under contract DE-AC02-06CH11357.

Conflicts of Interest: The authors declare no conflict of interest.

\section{Notes}

This independent sample can be constructed after inverting the quality cuts on the electromagnetic object reconstruction.

Such distributions are available as additional material from the ATLAS web page of the paper.

Due to the approximate nature of this study, we did not take into account systematic effects in the limit calculation. For two-jet plus lepton (or two lepton) invariant masses, systematic uncertainties for exclusion limits are expected to be dominated by jet energy scale and jet energy resolution. Typically, such experimental effects are not significant (i.e., within the 1-sigma band on the limits).

\section{References}

1. ATLAS Collaboration. Search for new phenomena in dijet mass and angular distributions from $p p$ collisions at $\sqrt{s}=13 \mathrm{TeV}$ with the ATLAS detector. Phys. Lett. 2016, b754, 302-322. [CrossRef]

2. ATLAS Collaboration. Search for new phenomena in dijet events using $37 \mathrm{fb}^{-1}$ of $p p$ collision data collected at $\sqrt{s}=13 \mathrm{TeV}$ with the ATLAS detector. Phys. Rev. 2017, D96, 052004. [CrossRef]

3. CMS Collaboration. Search for dijet resonances in proton-proton collisions at $13 \mathrm{TeV}$ and constraints on dark matter and other models. Phys. Lett. 2017, b769, 520-542. [CrossRef]

4. CMS Collaboration. Search for narrow resonances in dijet final states at $\sqrt{(s)}=8 \mathrm{TeV}$ with the novel CMS technique of data scouting. Phys. Rev. Lett. 2016, 117, 031802. [CrossRef] 
5. CMS Collaboration. Search for narrow resonances in the b-tagged dijet mass spectrum in proton-proton collisions at $\sqrt{s}=8 \mathrm{TeV}$. Phys. Rev. Lett. 2018, 120, 201801. [CrossRef]

6. CMS Collaboration. Search for Three-Jet Resonances in $p p$ Collisions at $\sqrt{s}=7$ TeV. Phys. Lett. 2012, 718, 329-347. [CrossRef]

7. CMS Collaboration. Search for pair-produced three-jet resonances in proton-proton collisions at $\sqrt{s}=13 \mathrm{TeV}$. Phys. Lett. 2019, 99, 012010. [CrossRef]

8. Accomando, E.; Coradeschi, F.; Cridge, T.; Fiaschi, J.; Hautmann, F.; Moretti, S.; Shepherd-Themistocleous, C.; Voisey, C. Production of Z'-boson resonances with large width at the LHC. Phys. Lett. 2020, 803, 135293. [CrossRef]

9. Li, Y.Y.; Nicolaidou, R.; Paganis, S. Exclusion of heavy, broad resonances from precise measurements of $W Z$ and $V H$ final states at the LHC. Eur. Phys. J. 2019, 79, 348. [CrossRef]

10. ATLAS Collaboration. Search for low-mass resonances decaying into two jets and produced in association with a photon using $p p$ collisions at $\sqrt{s}=13 \mathrm{TeV}$ with the ATLAS detector. Phys. Lett. 2019, 795, 56-75. [CrossRef]

11. CMS Collaboration. Search for Low-Mass Quark-Antiquark Resonances Produced in Association with a Photon at $\sqrt{s}=13$ TeV. Phys. Rev. Lett. 2019, 123, 231803. [CrossRef] [PubMed]

12. Sirunyan, A.M.; Tumasyan, A.; Adam, W.; Ambrogi, F.; Asilar, E.; Bergauer, T.; Brstetter, J.; Brondolin, E.; Dragicevic, M.; Erö, J.; et al. Search for low mass vector resonances decaying into quark-antiquark pairs in proton-proton collisions at $\sqrt{s}=13$ TeV. JHEP 2018, 1, 1-41. [CrossRef]

13. CMS Collaboration. Search for Low Mass Vector Resonances Decaying to Quark-Antiquark Pairs in Proton-Proton Collisions at 13 TeV. Phys. Rev. Lett. 2017, 119, 111802. [CrossRef] [PubMed]

14. CMS Collaboration. Search for low-mass resonances decaying into bottom quark-antiquark pairs in proton-proton collisions at $\sqrt{s}=13$ TeV. Phys. Rev. 2019, 99, 012005. [CrossRef]

15. CMS Collaboration. Search for low mass vector resonances decaying into quark-antiquark pairs in proton-proton collisions at $\sqrt{s}=13$ TeV. Phys. Rev. 2019, 100,1-41. [CrossRef]

16. Dorigo, T. Hadron Collider Searches for Diboson Resonances. Prog. Part. Nucl. Phys. 2018, 100, 211-261. [CrossRef]

17. ATLAS Collaboration. Search for dijet resonances in events with an isolated charged lepton using $\sqrt{s}=13$ TeV proton-proton collision data collected by the ATLAS detector. JHEP 2020, 6, 151. [CrossRef]

18. Sjöstr, T.; Mrenna, S.; Skands, P. PYTHIA 6.4 Physics and Manual. JHEP 2006, 5, 26.

19. Sjöstrand, T.; Mrenna, S.; Skands, P. A Brief Introduction to PYTHIA 8.1. Comput. Phys. Commun. 2008, 178, 852-867. [CrossRef]

20. Ball, R.D.; Bertone, V.; Carrazza, S.; Deans, C.S.; Del Debbio, L.; Forte, S.; Guffanti, A.; Hartl, N.P.; Latorre, J.I.; Rojo, J.; Ubiali, M. Parton distributions for the LHC Run II. JHEP 2015, 4, 40. [CrossRef]

21. Buckley, A.; Ferro, J.; Lloyd, S.; Nordström, K.; Page, B.; Rüfenacht, M.; Schönherr, M.; Watt, G. LHAPDF6: Parton density access in the LHC precision era. Eur. Phys. J. 2015, 75, 1-20. [CrossRef]

22. Cacciari, M.; Salam, G.P.; Soyez, G. The anti- $k_{t}$ jet clustering algorithm. JHEP 2008, 4, 63. [CrossRef]

23. Matteo, C.; Gavin, S.P.; Gregory, S. FastJet User Manual. Eur. Phys. J. 2012, C72, 1896. [CrossRef]

24. Chekanov, S.V.; Childers, J.T.; Proudfoot, J.; Wang, R.; Frizzell, D. Precision searches in dijets at the HL-LHC and HE-LHC. J. Instrum. 2018, 13, P05022. [CrossRef]

25. ATLAS Collaboration. Search for Quantum Black Hole Production in High-Invariant-Mass Lepton+Jet Final States Using $p p$ Collisions at $\sqrt{s}=8 \mathrm{TeV}$ and the ATLAS Detector. Phys. Rev. Lett. 2014, 112, 091804. [CrossRef]

26. Agashe, K.S.; Collins, J.H.; Du, P.; Hong, S.; Kim, D.; Mishra, R.K. LHC Signals from Cascade Decays of Warped Vector Resonances. JHEP 2017, 5, 78. [CrossRef]

27. Mikael, C.; Michael, S. Behavior of composite resonances breaking lepton flavor universality. Phys. Rev. 2018, 98, 035010. [CrossRef]

28. Alwall, J.; Frederix, R.; Frixione, S.; Hirschi, V.; Maltoni, F.; Mattelaer, O.; Shao, H.-S.; Stelzer, T.; Torrielli, P.; Zaro, M. The automated computation of tree-level and next-to-leading order differential cross sections, and their matching to parton shower simulations. JHEP 2014, 7, 79. [CrossRef]

29. Chekanov, S.V. HepSim: a repository with predictions for high-energy physics experiments. Adv. High Energy Phys. 2015, 2015, 136093. [CrossRef]

30. Cowan, G.; Cranmer, K.; Gross, E.; Vitells, O. Asymptotic formulae for likelihood-based tests of new physics. Eur. Phys. J. 2011, 71, 1554. [CrossRef] 